\title{
Controlled Variable Oxidative Doping of Individual Organometallic Nanoparticles
}

\author{
A Feng ${ }^{\mathrm{a}}$, W Cheng ${ }^{\mathrm{a}}$, J. Holter ${ }^{\mathrm{b}}$, N. Young ${ }^{\mathrm{b}}$, R G. Compton ${ }^{\mathrm{a} *}$
}

\begin{abstract}
The charging and controlled oxidative doping of single organometallic ferrocene nanoparticles is reported in aqueous sodium tetrafluoroborate using the nano-impacts method. It is shown that ferrocene nanoparticles of ca. 105 nm diameter are essentially quantitatively oxidatively doped with the uptake of one tetrafluoroborate anion per ferrocene molecule at suitably high overpotentials. By using lower potentials it is possible to achieve low doping levels of single nanoparticles in a controlled manner.
\end{abstract} and insertion of ions from the nanoparticulate matrix

\section{Introduction}

and is conventionally studied as an ensemble modified on an electrode surface. Studying the charging and doping of individual nanoparticles is challenging, because the accompanying charge transfer is strongly coupled to mass transport processes which typically can only be averaged when using an ensemble of nanoparticles. Desirably individual nanoparticles can become fully charged / discharged.

Nanoparticles are widely used in many applications such as catalysis, energy conversion for batteries and fuel cells ${ }^{[1]}$. The underpinning process of these applications is charge transfer involving the extraction

[a] Ms A Feng, Dr. W Cheng, Prof. Dr. R G. Compton*

Department of Chemistry, Physical \& Theoretical Chemistry Laboratory, Oxford

University, South Parks Road, Oxford, OX1 3QZ, United Kingdom

*Corresponding email: richard.compton@chem.ox.ac.uk

[b] Dr. J. Holterb, Dr. N. Young, Department of Materials, Oxford University,

Parks Road, Oxford, OX1 3PH, UK

Supporting information for this article is available on the WWW under

http://dx.doi.org/10.1002/chem.2016xxxxx
The method of "nano impacts" has been recently developed to study single nanoparticles when they randomly collide, by virtue of their Brownian motion, with a potentiostated electrode. If the electrode is potentiostated at a suitable potential this can induce oxidation/reduction of, or catalytic reaction by, the nanoparticle ${ }^{[2]}$. Hitherto, the nano-impacts method has been applied to characterise nanoparticles including metal $^{[3]}$, metal oxides ${ }^{[4]}$, metal dichalcogenides ${ }^{[5]}$ and sulfide $^{[6]}$, polymeric ${ }^{[7]}$, organic nanoparticles ${ }^{[8]}$, and 
macromolecules ${ }^{[9]}$, and extended to soft nanoparticles such as liposomes ${ }^{[10]}$, vesicles ${ }^{[11]}$, viruses $^{[12]}$, micelles $^{[13]}$, droplets ${ }^{[14]}$. In these experiments, charge transfer leads to complete dissolution of the nanoparticles $^{[3 \mathrm{a}, 8 \mathrm{a}, 14 \mathrm{a}]}$, or partial electrolysis to produce core-shell nanoparticles ${ }^{[9 b]}$.

Although the solution electrochemistry of ferrocenelike organometallic compounds is well studied, the electron transfer associated with ion transport from/to aqueous insoluble organometallic compounds has yet to be fully unravelled ${ }^{[15]}$. In particular, the charging and controlled doping of organometallic based nanoparticle phases have not been previously reported although recently there is significant interest for design and synthesis of organometallic nanomaterials as new materials ${ }^{[16]}$ and for diverse catalytic applications ${ }^{[17]}$. In the present paper, the charging and controlled oxidative doping of single ferrocene nanoparticles is reported in aqueous sodium tetrafluoroborate using the nano-impacts method ${ }^{[2]}$. It will be shown that ferrocene nanoparticles of ca. 105 nm diameter are essentially quantitatively oxidatively doped with the uptake of one tetrafluoroborate anion per ferrocene molecule at suitably positive potentials. With the applications of lower potentials partial doping can be realised in a controlled manner at the single nanoparticle level.

\section{Results and Discussion}

An emulsified mediated evaporation method was previously reported to form ferrocene nanoparticles as colloids in water ${ }^{[18]}$. A facile effective re-precipitation method is used for the synthesis of aqueous dispersion of ferrocene nanoparticles ${ }^{[19]}$. The particles are characterised by both SEM and DLS (see Experimental Section). The shape of the particles is shown to be quasi-spherical, with a mean diameter of 104.6 \pm 49.9 , which are similar to those from DLS estimation (Figure 1).

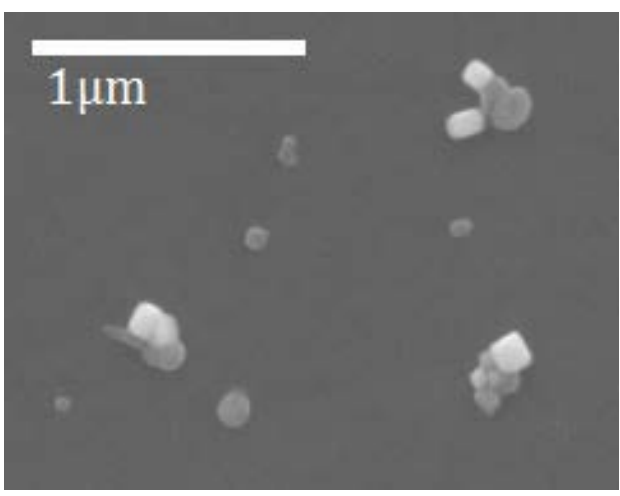

(a) 


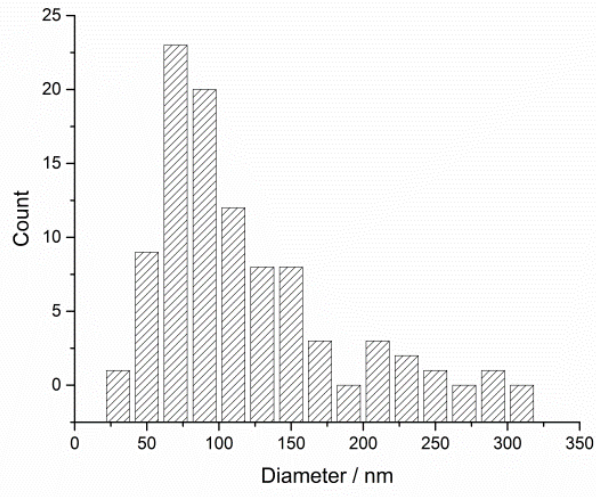

(b)

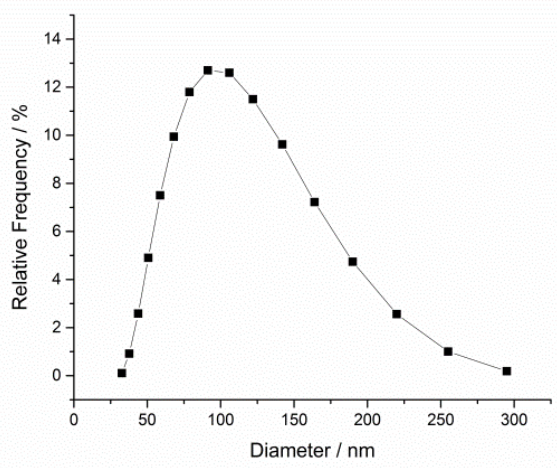

(c)

Figure 1 (a) SEM image of the synthesised Fc nanoparticles by nanoprecipitation, and the size distribution of synthesised Fc nanoparticles measured by SEM (b) and DLS (c).

Cyclic voltammetry was applied to study the oxidative doping of an ensemble of ferrocene nanoparticles drop-cast on the electrode. The nano-impacts method was then applied to characterise the oxidative doping of single ferrocene nanoparticles.

First, to study the redox properties of the ensemble of ferrocene nanoparticles, cyclic voltammetry was recorded for a ferrocene nanoparticle modified EPPG electrode made by the drop casting method (see Experimental) in three different $0.10 \mathrm{M}$ aqueous electrolytes: $\mathrm{NaCl}, \mathrm{NaBF}_{4}, \mathrm{NaClO}_{4}$, at a scan rate of 25 $\mathrm{mV} \mathrm{s}^{-1}$ as shown in Figure 2. In Figure 2 (a), it can be seen that the cyclic voltammetry of oxidation of ferrocene nanoparticles shows a similar shape in all three electrolytes with oxidation peak potentials of $0.27 \mathrm{~V}, 0.28 \mathrm{~V}, 0.29 \mathrm{~V}$ in $\mathrm{NaCl}, \mathrm{NaClO}_{4}, \mathrm{NaBF}_{4}$ and back peaks at $0.12 \mathrm{~V}, 0.14 \mathrm{~V}, 0.12 \mathrm{~V}$ respectively. The back peaks for all the three electrolytes may be attributed to a reduction process for oxidised ferrocene nanoparticles. The existence of back peaks implies there is doping of ferrocene nanoparticles on oxidation, rather than dissolution of the particle as molecular ferrocenium cation. The oxidation and back peaks were also seen for crystalline ferrocene (see SI), with similar oxidation peak potentials of $0.30 \mathrm{~V}, 0.31 \mathrm{~V}$, $0.32 \mathrm{~V}$ in $\mathrm{NaCl}, \mathrm{NaClO}_{4}, \mathrm{NaBF}_{4}$ and back peaks at $0.17 \mathrm{~V}, 0.17 \mathrm{~V}, 0.19 \mathrm{~V}$ at a scan rate of $25 \mathrm{mV} \mathrm{s}^{-1}$ respectively. The slight difference of oxidative and reductive peak potentials in the electrolytes for dropcast ferrocene nanoparticles or crystalline ferrocene is likely due to doping propensity of various anions already reported in microcrystalline decamethylferrocene ${ }^{[20]}$, where the doping of various anions was found to be considerably dependent on the hydrophobicity of the electrolyte anion for 
microcrystalline decamethylferrocene ${ }^{[20]}$. Note that differences between microcrystals as reported in the literature and amorphous nanoparticles are likely expected.

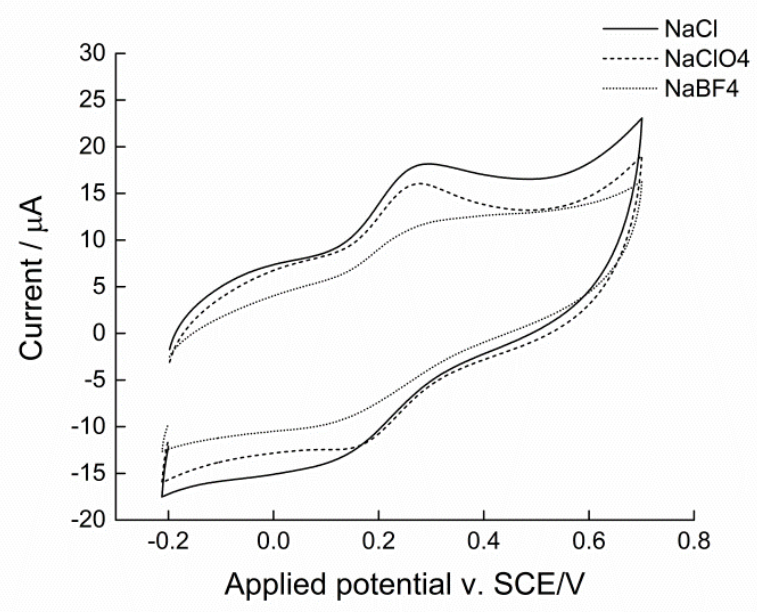

(a)

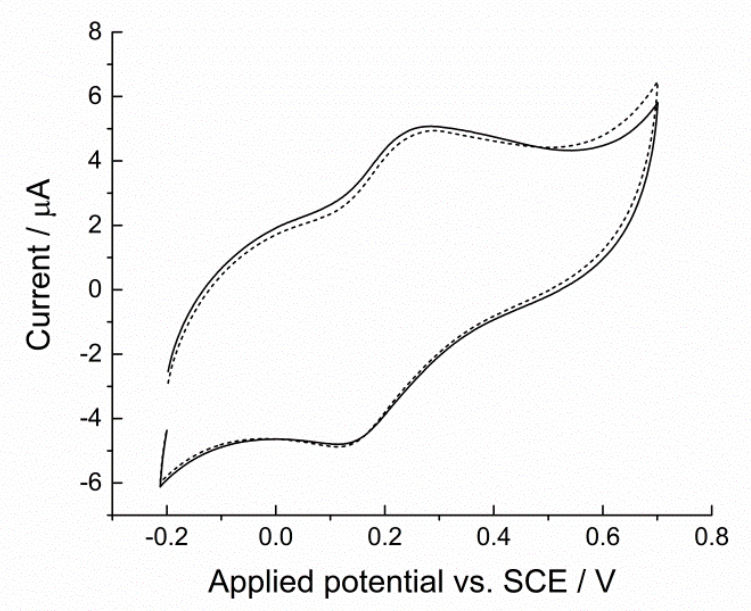

(b)

Figure 2 Cyclic voltammetric response of a Fc nanoparticle modified EPPG electrode (a) in three $0.1 \mathrm{M}$ aqueous electrolytes at a scan rate of 100 $\mathrm{mV} \mathrm{s}^{-1}$ (solid line: $\mathrm{NaCl}$, dashed line: $\mathrm{NaClO}_{4}$, dotted line: $\mathrm{NaBF}_{4}$ ); (b) for the first and second scans in $0.1 \mathrm{M} \mathrm{NaBF}_{4}$ at a scan rate of $25 \mathrm{mV} \mathrm{s}^{-1}$.

Figure 2(b) shows the first and second scans of the oxidative doping of ferrocene nanoparticles modified on an EPPG electrode in $0.1 \mathrm{M} \mathrm{NaBF}_{4}$. Again, the significant oxidation peak at ca. $+0.27 \mathrm{~V}$ in the first scan corresponds to the ferrocene oxidation on the surface of the electrode, while the second scan then reveals a near superposition of the first scan, which indicates there was nearly the exact charge passed during oxidation and reduction between the two consecutive scans.. Therefore, it suggests that the oxidative doping of ferrocene nanoparticles shows little loss of oxidised ferrocene nanoparticles and a chemically reversible process which likely involves the transition between ferrocene nanoparticles to ferrocenium nanoparticles on the surface of the electrode, as shown in Scheme 1.

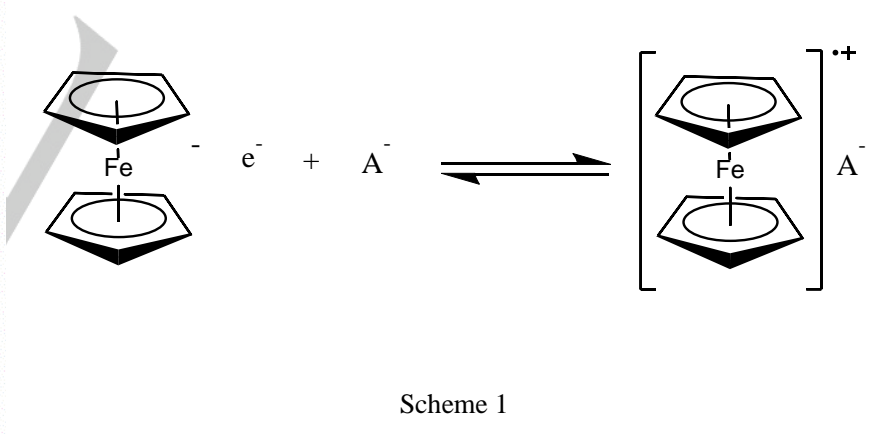

The charge from the oxidative doping process can be obtained from the integration of the peak from the first scan as $1.9 \times 10^{-5} \mathrm{C}$, corresponding to about $16 \%$ oxidation of drop-cast ferrocene nanoparticles estimated if assuming the oxidative doping of ferrocene nanoparticles undergoes a one electron transfer with the uptake of one tetrafluoroborate anion 
per single ferrocene molecule unit. The incomplete oxidative doping may be due to the aggregation of ferrocene nanoparticles where only the nanoparticles at the periphery of the aggregated particles that are in contact with the electrode are oxidised ${ }^{[21]}$.

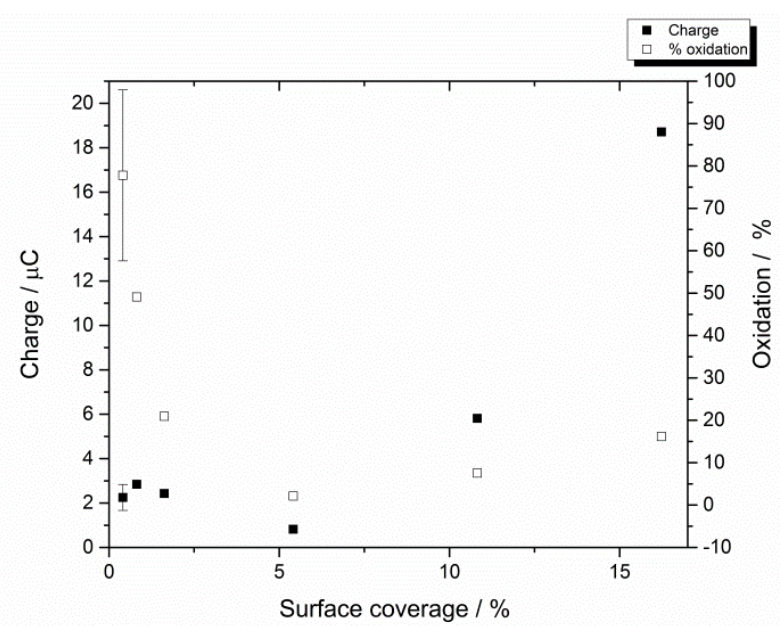

Figure 3 Oxidative charge (black squares) and percentage of ferrocene nanoparticles oxidised (hollow squares) derived from cyclic voltammetric response of a Fc nanoparticles modified EPPG electrode in $0.1 \mathrm{M} \mathrm{NaBF}_{4}$ electrolyte at a scan rate of $25 \mathrm{mV} \mathrm{s}^{-1}$ versus surface coverage.

Different loadings (converted to the percentage of surface coverage assuming that there is a monolayer of ferrocene nanoparticles drop-cast on the electrode surface when the surface coverage is $100 \%$, see SI) of ferrocene nanoparticles were drop cast on the electrode and the oxidation peak charge was found to increase with higher loadings (Figure 3), while the percentage of oxidised ferrocene nanoparticles was found to increase with decreased surface coverage, further suggesting that the oxidation of the ensemble of ferrocene nanoparticles largely depends on the state of agglomeration/aggregation of nanoparticles. In particular, when the surface coverage of ferrocene nanoparticles was reduced to $0.4 \%$, near complete oxidative doping $(78 \% \pm 20 \%)$ was likely seen (Figure 3). Further quantitative information from the oxidative doping of ferrocene nanoparticles is found difficult to be elucidated from the ensemble of nanoparticles dropcast on the electrode as the oxidative doping of nanoparticles ensembles reflect the aggregation state of nanoparticles $^{[21 a]}$, overlapping of adjacent diffusion layers $^{[22]}$, as well as the charge insertion of ferrocene nanoparticles at the solid/solution (electrolyte) boundary $^{[23]}$. Therefore in the next section nano-impact experiments are used to further provide quantitative information of the oxidative doping of single individual ferrocene nanoparticles.

To study the oxidative doping of single ferrocene nanoparticles, a clean, unmodified carbon microelectrode was placed in $0.1 \mathrm{M}$ sodium tetrafluoroborate solution and a known concentration of ferrocene nanoparticles suspension added. The oxidative potential was selected to be $+0.5 \mathrm{~V}$ vs. SCE, a sufficiently high potential to ensure complete oxidation of ferrocene nanoparticles, as discussed above. Under potentiostatted conditions, clear oxidative spikes from individual ferrocene nanoparticles were observed (Figure 4). The onset of 
these Faradaic spikes was found to be dependent on oxidative potentials and no spike was observed at lower oxidation potentials of $+0 \mathrm{~V}$ or below, showing that these spikes result from the oxidative doping of ferrocene NPs. A control experiment was conducted at the potential of $+0.5 \mathrm{~V}$ vs. SCE with no ferrocene nanoparticles in the solution, and no spikes were seen, confirming that the individual spikes is due to the random collisions of single ferrocene nanoparticles with the surface of the electrode and subsequent oxidative doping and electron transfer from the electrode.

Figure 4(a) shows a typical chronoamperometric profile of oxidative Faradaic spikes of individual ferrocene nanoparticles at $+0.50 \mathrm{~V}$ vs. SCE. A total of 142 spikes were eventually observed from 40 recorded chronoamperograms of $5 \mathrm{~s}$ duration each, corresponding to the oxidative doping of 142 individual ferrocene nanoparticles. The charge transferred during the oxidative doping of single ferrocene nanoparticles was calculated by integrating the individual spikes area (Figure 4(b)). To quantify the oxidative doping of single ferrocene nanoparticles, the mean diameter $d$ of ferrocene nanoparticles was estimated to be $105 \pm 50 \mathrm{~nm}$ through SEM (Figure 1). Assuming complete oxidative doping of single ferrocene nanoparticles, the theoretical charge $(Q t)$ of electron transfer can be determined by Equation 1, knowing that ferrocene nanoparticles are quasispherical.

$$
Q=\frac{d^{3} n F \pi \rho}{6 M}
$$

Equation 1

where $\mathrm{d}$ is the diameter of ferrocene nanoparticles, $\mathrm{M}$ is the molar mass of ferrocene and $\rho$ is the density of crystalline ferrocene (the values are given in SI) and F is the Faraday constant. The parameter $\mathrm{n}$ is the number of electrons transferred per ferrocene molecule during oxidative doping, where $\mathrm{n}=1$, as discussed above.

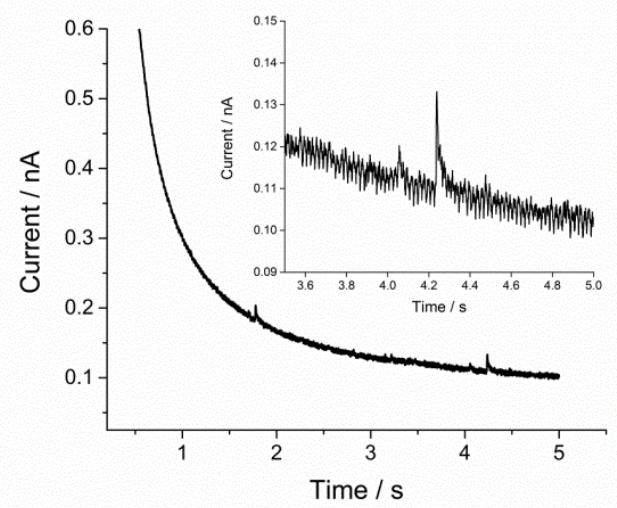

(a)

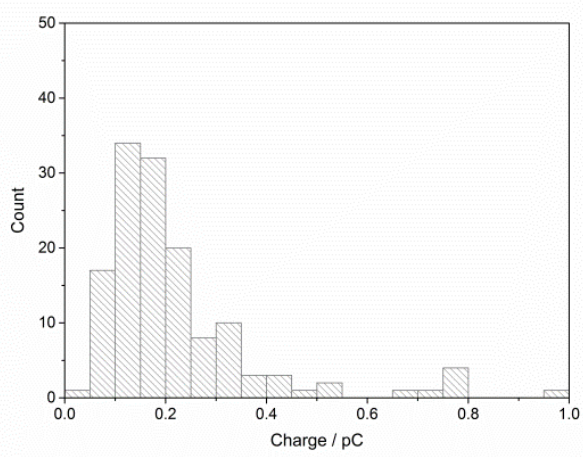

(b)

Figure 4 (a) Chronoamperometric profiles showing oxidative Faradaic spikes of Fc nanoparticles in $0.1 \mathrm{M}$ sodium tetrafluoroborate at $+0.50 \mathrm{~V}$ vs SCE. The inset showing the detailed impact spikes. (b) The distribution of charge from oxidative doping of single Fc nanoparticles by analysis of the 142 oxidation spikes from nano-impact experiments. 
Comparing the average charge from oxidative doping of 142 individual ferrocene nanoparticles $Q_{y}\left(Q_{y}=0.37\right.$ $\pm 0.09 \mathrm{pC}$ ) from experimental nano-impacts with the theoretical estimated charge $Q_{t}\left(Q_{t}=0.35 \mathrm{pC}\right)$ (Equation 1) assuming the complete doping of ferrocene nanoparticle with diameter $105 \mathrm{~nm}$ indicates that there is near-complete oxidative doping of single ferrocene nanoparticles at the oxidative potential of 0.5 V.

The ferrocene nanoparticles are quantitatively oxidised with the uptake of one tetrafluoroborate anion per ferrocene molecule, as illustrated in Scheme 1. Considering size distribution of the synthesised ferrocene nanoparticles, the distribution of charge from the oxidative doping of individual ferrocene nanoparticles by nano-impacts of all 142 single ferrocene nanoparticles was also compared to the theoretical estimation of ferrocene nanoparticles seen by SEM as analysed from the above equation, further indicating near-complete oxidative doping of ferrocene nanoparticles when recognising the size distribution of the analysed nanoparticles (Figure 5).

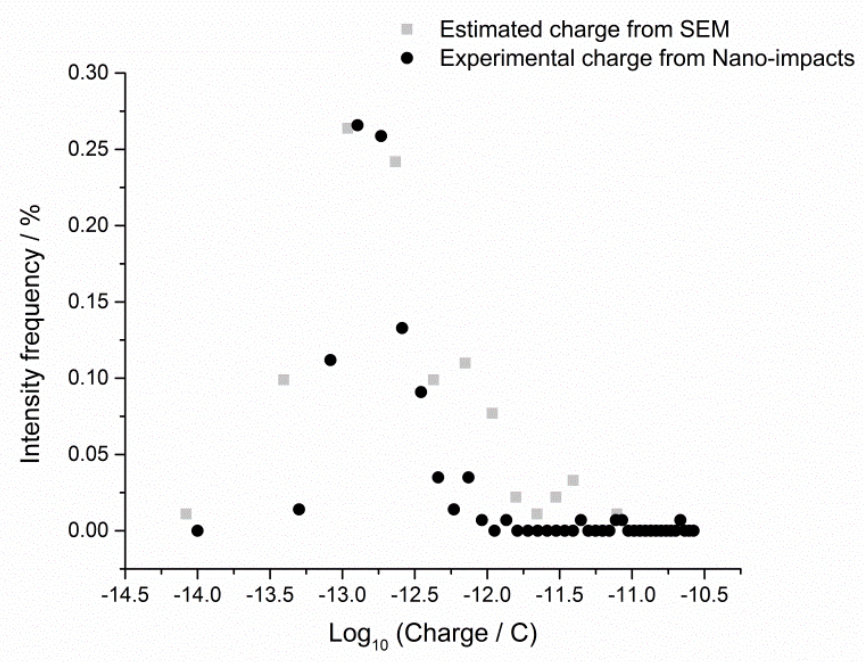

Figure 5 Histogram shows the charge distribution of single Fc nanoparticles derived from charge per spike from nano-impact experiments and the theoretical estimated from SEM data (Figure 1) according to Equation 1.

Finally and novelly, the oxidative doping of single ferrocene nanoparticles at different oxidative potentials was investigated to explore the possibility of potential controlled partial doping. The charge of Faradaic spikes resulting from the impacting of ferrocene nanoparticles was found to be dependent on the oxidative potentials. 


\section{Conclusions}

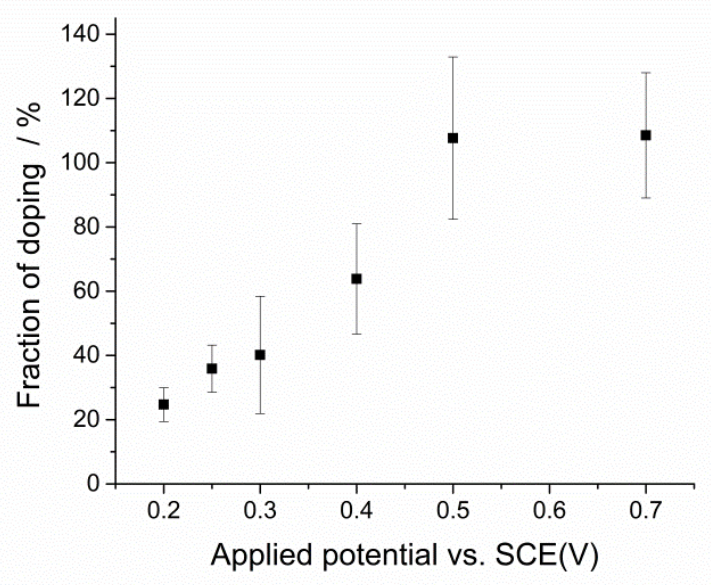

Figure 6 Fraction of oxidative doping of single ferrocene nanoparticles vs. the oxidative potential used for the nano impacts studies.

By comparing the average oxidative charge $Q_{y}$ resulting from the oxidative doping of all the measured individual nanoparticles from experimental nanoimpacts at different oxidative potentials against the ideal charge $Q i$, the fraction of oxidative doping of single ferrocene nanoparticle $\left(y=Q_{y} / Q i\right)$ can be determined, as shown in Figure 6. The quantitative analysis of the fraction of oxidative doping for single ferrocene nanoparticle suggests the dependence of doping of single nanoparticles on the applied potentials. The result show controlled variable doping and quantitative analysis of individual ferrocene nanoparticles is viable, and provides new quantitative information for the customised design and controlled synthesis of organometallic nanoparticles.
The charging and controlled oxidative doping of single ferrocene nanoparticles is reported in aqueous sodium tetrafluoroborate using the nano-impacts method. It is shown that the nanoparticles of ca. $105 \mathrm{~nm}$ diameter are essentially quantitatively oxidatively doped with the uptake of one tetrafluoroborate anion per ferrocene molecule if a sufficiently high overpotential ( $>\sim 0.5 \mathrm{~V}$ vs. SCE) is used to oxidise the particles. If lower potentials are used then controlled partial doping of single nanoparticles is realised validating a simple strategy to synthesise and characterise doped organometallic nanoparticles at the individual nanoparticle level.

\section{Experimental Section}

\section{Materials}

Ferrocene (Fc, 98 \%) and acetonitrile (HPLC, 99.9 \%) were purchased from Sigma-Aldrich and used as received. All solutions were made up with deionised water of resistivity not less than $18.2 \mathrm{M} \Omega \mathrm{cm}$ (Millipore) at $298 \mathrm{~K}$ and degassed with nitrogen (oxygen-free, BOC Gases plc) before use. 
Synthesis of ferrocene nanoparticles

Briefly, ferrocene was dissolved in acetonitrile to a concentration of $10 \mathrm{mM} .800 \mu \mathrm{L}$ of ferrocene solution was then added dropwise over 30 seconds to into 20 $\mathrm{mL}$ de-ionized water under vigorous stirring by magnetic vortexing at $800 \mathrm{rpm}$. They were then subjected to sonication for $5 \mathrm{~min}$ at room temperature. Synthesis was optimised to produce a narrow, reproducible size distribution.

Characterisation of ferrocene nanoparticles

Dynamic Light Scattering (DLS, Malvern Instruments Ltd, UK) was used to characterise the size distribution of the synthesised NPs (Figure 1). Scanning Electron Microscopy (SEM) was conducted on a dropcast of the nanoparticles on a glassy carbon substrate with a Jeol 6500F SEM (Jeol, Japan). Operating at an accelerating voltage of $5 \mathrm{kV}$ and using the secondary electron image, the size distribution and particle shape could be determined.

\section{Cyclic voltammetry of ferrocene nanoparticles}

Drop casting was used to modify an edge plane pyrolytic graphite (EPPG) electrode with a film of nanoparticles. This method involves placing a drop of nanoparticle suspension on the surface of the electrode followed by evaporation of the solvent. In the experiment, different amounts of freshly prepared nanoparticle suspensions were dropped on the surface of an EPPG electrode (the different surface coverages are given in the SI), then the electrode was gently blown with nitrogen to evaporate the solvent. Cyclic voltammetry was subsequently used for electrochemical studies with the modified electrode in three electrolytes.

Electrochemical experiments were conducted at $25^{\circ} \mathrm{C}$ with a double Faraday cage with a three electrode system using an Autolab II potentiostat (MetrohmAutolab BV, Netherlands). For cyclic voltammetry measurements, an EPPG electrode (diameter $=4.9$ $\mathrm{mm}$ ) was used as the working electrode, a saturated calomel electrode (SCE) as the reference electrode and a platinum foil as the counter electrode. The EPPG electrode was polished before experiments on alumina powder on a cloth pad in the size sequence $1.0 \mu \mathrm{m}, 0.3$ $\mu \mathrm{m}, 0.05 \mu \mathrm{m}$, then sonicated to remove alumina. Experiments were thermostatted to $25^{\circ} \mathrm{C}$. Additional data analysis and curve fitting was made using GPES 
4.9, Nova 1.11 and Origin Pro 9 (Origin Lab partly funding this work. We thank Dr. D Omanović

Corporation).

Nanoimpacts of ferrocene nanoparticles

For the electrochemical study of single nanoparticles, chronoamperometry was performed with a carbon microdisc electrode $($ diameter $=11 \mu \mathrm{m})$ as working electrode and the same reference and counter electrodes as used for macroelectrode experiments in 0.1 M sodium tetrafluoroborate with a known amount of nanoparticles added. The carbon micro electrode diameter was calibrated electrochemically using the steady-state voltammetry of $1.0 \quad \mathrm{mM}$ Hexaammineruthenium (III) chloride in $0.1 \mathrm{M} \mathrm{KCl}$. The in-house software "Signal Counter" developed by Dr. D Omanović (Center for Marine and Environmental Research Zagreb, Croatia) ${ }^{[18]}$ was used for analysis of the impact spikes observed and OriginPro 9 for statistical analysis of the charge passed per spike.

\section{Acknowledgements}

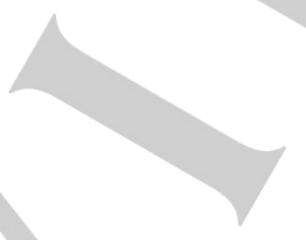

The European Research Council under the European

Union's Seventh Framework Programme (ERC Grant Agreement n. 320403) is gratefully acknowledged for
(Center for Marine and Environmental Research Zagreb, Croatia) for developing the Signal Counter software for data analysis. We would also like to thank Dr Blake Plowman for helpful discussions on SEM characterisation of ferrocene nanoparticles.

\section{Keywords:}

Controlled Doping • Organometallic Nanoparticles • Electrochemistry $\bullet$ Nanoimpacts

\section{Reference}

a) Q. F. Zhang, E. Uchaker, S. L. Candelaria, G. Z. Cao, Chem Soc Rev 2013, 42, 3127-3171; b) F. Zaera, Chem Soc Rev 2013, 42, 2746-2762.

[2] a) M. Pumera, Acs Nano 2014, 8, 7555-7558; b) W. Cheng, R. G. Compton, Trac-Trend Anal Chem 2014, 58, 79-89; c) N. V. Rees, Electrochem Commun 2014, 43, 83-86; d) A. J. Bard, H. J. Zhou, S. J. Kwon, Isr J Chem 2010, 50, 267-276; e) X. Y. Xiao, F. R. F. Fan, J. P. Zhou, A. J. Bard, J Am Chem Soc 2008, 130, 16669-16677; f) J. M. Kahk, N. V. Rees, J. Pillay, R. Tshikhudo, S. Vilakazi, R. G. Compton, Nano Today 2012, 7, 174-179; g) S. E. Fosdick, M. J. Anderson, E. G. Nettleton, R. M. Crooks, J Am Chem Soc 2013, 135, 5994-5997; h) R. Dasari, D. A. Robinson, K. J. Stevenson, J Am Chem Soc 2013, 135, 570-573.

[3] a) Y. G. Zhou, N. V. Rees, R. G. Compton, Angew Chem Int Edit 2011, 50, 4219-4221; b) M. Giovanni, A. Ambrosi, Z. Sofer, M. Pumera, Electrochem Commun 2015, 56, 16-19.

[4] K. Tschulik, B. Haddou, D. Omanovic, N. V. Rees, R. G. Compton, Nano Res 2013, 6, 836841.

[5] C. S. Lim, S. M. Tan, Z. Sofer, M. Pumera, Acs Nano 2015, 9, 8474-8483.

[6] C. S. Lim, M. Pumera, Phys Chem Chem Phys 2015, 17, 26997-27000.

[7] X. F. Zhou, W. Cheng, R. G. Compton, Angew Chem Int Edit 2014, 53, 12587-12589. 
[8] a) W. Cheng, X. F. Zhou, R. G. Compton, Angew Chem Int Edit 2013, 52, 12980-12982; b) X. F. Zhou, W. Cheng, R. G. Compton, Nanoscale 2014, 6, 6873-6878.

[9] a) J. E. Dick, C. Renault, A. J. Bard, J Am Chem Soc 2015, 137, 8376-8379; b) X. F. Zhou, W. Cheng, R. G. Compton, Nanoscale 2015, 7, 15719-15726; c) E. J. E. Stuart, K. Tschulik, C. Batchelor-McAuley, R. G. Compton, Acs Nano 2014, 8, 7648-7654.

[10] W. Cheng, R. G. Compton, Angew Chem Int Edit 2014, 53, 13928-13930.

[11] J. Dunevall, H. Fathali, N. Najafinobar, J. Lovric, J. Wigstrom, A. S. Cans, A. G. Ewing, J Am Chem Soc 2015, 137, 4344-4346.

[12] J. E. Dick, A. T. Hilterbrand, A. Boika, J. W. Upton, A. J. Bard, P Natl Acad Sci USA 2015, 112, 5303-5308.

[13] H. S. Toh, R. G. Compton, Chem Sci 2015, 6, 5053-5058.

[14] a) B. K. Kim, J. Kim, A. J. Bard, J Am Chem Soc 2015, 137, 2343-2349; b) B. K. Kim, A. Boika, J. Kim, J. E. Dick, A. J. Bard, J Am Chem Soc 2014, 136, 4849-4852; c) W. Cheng, R. G. Compton, Angew Chem Int Edit 2015, 54, 7082-7085.

[15] a) K. Uosaki, Y. Sato, H. Kita, Langmuir 1991, 7, 1510-1514; b) T. Kondo, M. Okamura, K. Uosaki, J Organomet Chem 2001, 637, 841844. c) A. M. Bond, F. Scholz, Langmuir 1991, 7, 3197-3204.

[16] a) Z. M. Hudson, I. Manners, Science 2014, 344, 481-482; b) W. E. Geiger, Organometallics 2011, 30, 28-31.

[17] a) N. Yan, Y. Yuan, P. J. Dyson, Dalton T 2013, 42, 13294-13304; b) K. Philippot, Abstr Pap Am Chem S 2012, 243.

[18] N.Kaus, A. M. Collins, S. Mann, AIP Conf. Proc. 2014, 1621, 657.

[19] D. Horn, J. Rieger, Angew Chem Int Edit 2001, 40, 4331-4361.

[20] A. M. Bond, F. Marken, J Electroanal Chem 1994, 372, 125-135.

[21] a) S. J. Cloake, H. S. Toh, P. T. Lee, C. Salter, C. Johnston, R. G. Compton, Chemistryopen 2015, 4, 22-26; b) K. Ngamchuea, K. Tschulik, S. Eloul, R. G. Compton, Chemphyschem 2015, 16, 2338-2347; c) H. S. Toh, K. Jurkschat, R. G. Compton, Chem-Eur J 2015, 21, 2998-3004; d) H. S. Toh, C. Batchelor-McAuley, K. Tschulik, M. Uhlemann, A. Crossley, R. G. Compton, Nanoscale 2013, 5, 4884-4893.
[22] T. J. Davies, C. E. Banks, R. G. Compton, J Solid State Electr 2005, 9, 797-808.

[23] a) F. Scholz, S. Komorsky-Lovric, M. Lovric, Electrochem Commun 2000, 2, 112-118; b) S. Komorsky-Lovric, M. Lovric, F. Scholz, J Electroanal Chem 2001, 508, 129-137. 


\section{Full Paper}

The charging and controlled oxidative doping of single organometallic ferrocene nanoparticles is reported using the recently developed nanoimpacts method, showing controlled variable doping of individual ferrocene nanoparticles.
A Feng $^{\mathrm{a}}$, W Cheng ${ }^{\mathrm{a}}$, J. Holter ${ }^{\mathrm{b}}$, N. Young ${ }^{\mathrm{b}}, \mathrm{R}$

$$
\text { G. Compton }{ }^{\mathrm{a}^{*}}
$$

Page 1. - Page 11.

\section{Controlled Variable Oxidative Doping of} Individual Organometallic Nanoparticles
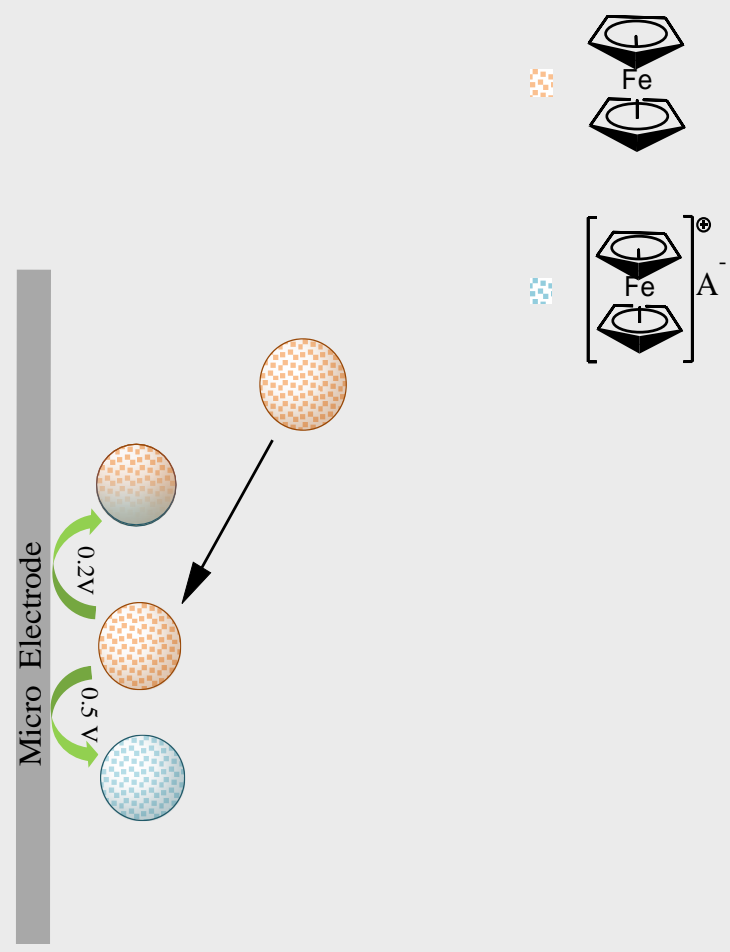